\title{
Uveal Melanoma Metastatic in the Liver
}

National Cancer Institute

\section{Source}

National Cancer Institute. Uveal Melanoma Metastatic in the Liver. NCI Thesaurus. Code C150130.

A melanoma that originates from melanocytes of the uveal tract and has spread to the liver. 